\title{
Using Topical Anesthesia for Standing Phacoemulsification Cataract Surgery and Intraocular Lens Implantation
}

\author{
Ronghan Wu, Qinxiang Zheng, Wensheng Li \\ Eye Hospital, Wenzhou Medical College, Wenzhou, China \\ Email: drlws@163.net
}

Received February 13, 2012; revised April 17, 2012; accepted April 26, 2012

\begin{abstract}
Introduction: To report by using topical anesthesia for standing phacoemulsification and intraocular lens implantation for two patients who need cataract extraction but unable to lie flat with marked cervical kyphosis due to long-standing ankylosing spondylitis and chronic uveitis. Methods: Two patients are unable to lie flat respectively during phacoemulsification underwent this technique in Jingdong and Tonghai county Hospital of Yunnan province in China. Each patient was positioned erect or semirecumbent in a standard reclining cataract surgical chair. The ceiling-mounted microscope was rotated 60 degrees from the vertical to point toward the patient. Results: The intraoperative and postoperative periods were uneventful in two patients, with good visual outcomes after surgery. Conclusion: This technique is valuable for situations where the patient requires upright positioning because of the inability to recline flat, and should be considered for cases where standard surgical positioning is not possible.
\end{abstract}

Keywords: Topical Anesthesia; Cataract Surgery; Intraocular Lens Implantation

\section{Introduction}

Patients unable to recline present a challenge to the cataract surgeon. Various diseases and conditions can cause deformities such as kyphosis or torticollis, making it difficult to position the patient with the head in the usual position. Other patients cannot recline because of chronic obstructive pulmonary disease or congestive heart failure. A few authors have reported techniques to work around these problems but they had encountered some complications [1,2]. In this paper, for the first time, we reported two cases of standing phacoemulsification and intraocular lens implantation by using topical anesthesia for two patients unable to lie flat, with good visual outcomes after surgery and no intraoperative and postoperative complications.

\section{Case Presentations}

\subsection{Case 1}

A 68-year-old woman had ankylosing spondylitis for the past 32 years. Since 1981, her mobility had deteriorated gradually with progression of her kyphosis, stiffness of the low back, and contractures of the hips and knees. She also had virtual fixation of hips in 45 degrees of flexion and adduction together with fixed flexion deformities in both knees. The patient had a long history of bilateral chronic uveitis, which had resulted in a phthisical left eye with no light perception. Visual acuity in the right eye was hand movements only with accurate light projection. Intraocular pressure, measured with a Tono-Pen as the patient could not be positioned at a slitlamp, was 24.5 $\mathrm{mmHg}$ in the right eye. She had a dense nuclear sclerotic cataract with extensive posterior synechiae, making the fundus examination impossible. Topical levobunolol was used for a long time according to her ophthalmic condition.

Topical anesthesia was applied using 0.5\% proparacaine hydrochloride (Alcaine, Alcon) three times before surgery. By raising the patient's feet and lowering the head end of the table, it was possible to lower the patient's head to an inclination of about 60 degrees from the horizontal. It was impossible to extend the patient's neck or to recline her further. This created an abnormal angle of approach for the operating microscope and the surgeon (W.S.L), who had to stand right facing the patient (Figure 1). The standing phacoemulsification technique consists of a standing surgeon, minimum magnifycation of surgical microscope (to avoid the use of the nondominant leg as much as possible for control of the pedal for the surgical microscope), and one-legged phacoemulsification technique (nondominant leg for body support and dominant leg for control of the phacoemulsification pedal). A clear corneal temporal tunnel incision was 


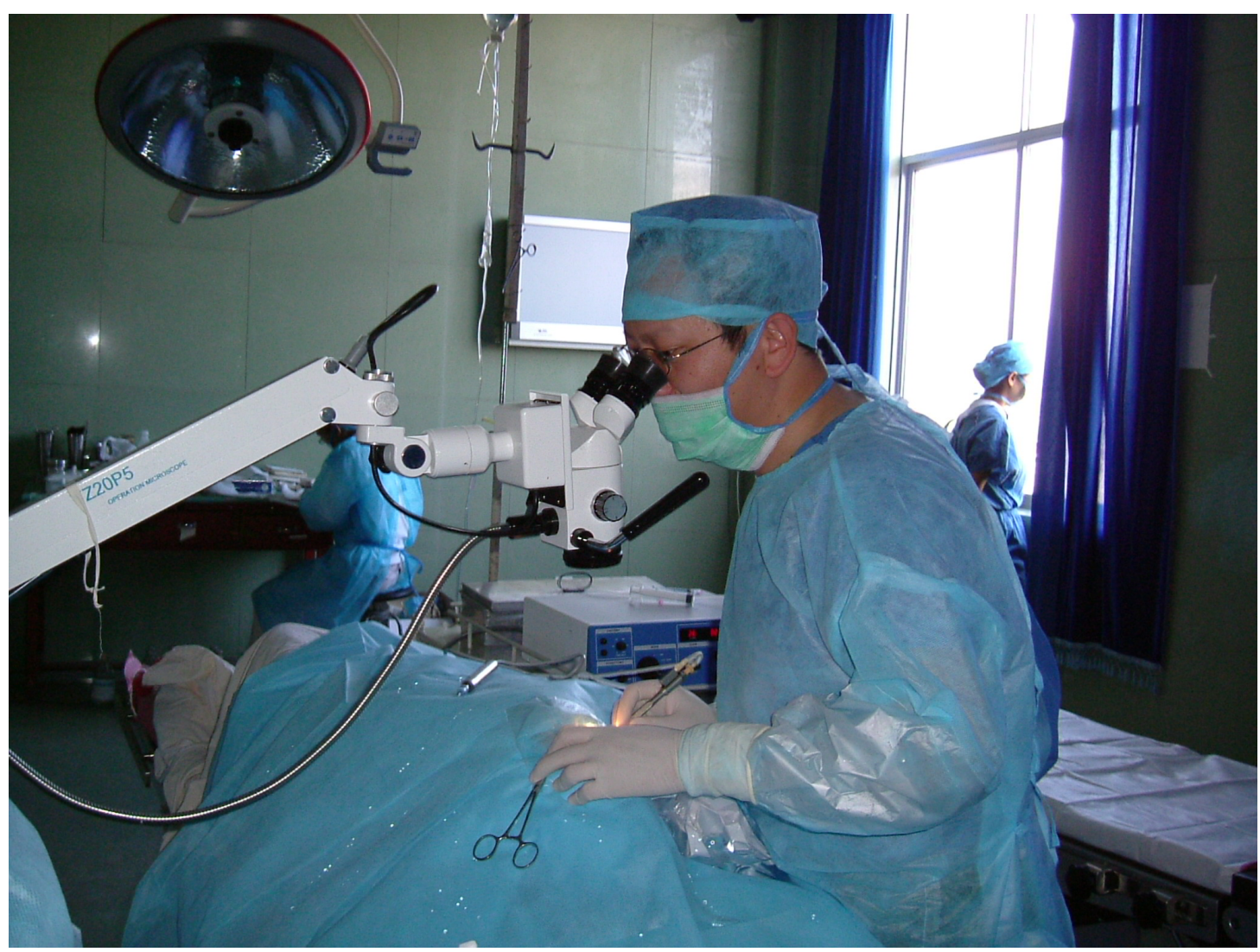

Figure 1. The standing phacoemulsification technique with the patient in reverse trendelenburg position. Position of the surgeon and operating microscope during surgery, showing the angle of approach.

made at 8 o'clock with a $3.0 \mathrm{~mm}$ disposable metal blade and a side port incision was made at 11 o'clock, sodium hyaluronate (DUOVISC, Alcon) was injected to stabilize the anterior chamber, capsular forceps were used to perform a continuous curvilinear capsulorhexis of $5.0 \mathrm{~mm}$ diameter, phacoemulsification was performed using a stop and chop technique. A +20.5D intraocular lens (OII, Ophthalmic Innovation International, INC) was inserted intracapsularly with $-0.80 \mathrm{D}$ residual degree. Effective phaco time was 3'20" with a mean of $21 \%$ of ultrasound power. The patient experienced with no discomfort during surgery and cooperated perfectly throughout the operation. Postoperatively, we suggest dilating surgery eyes once daily with short-acting agents, such as $1 \%$ tropicamide, rather than with long-acting agents, such as atropine. Topical corticosteroids were also used to help prevent formation of synechiae and minimize the inflammation. On the first postoperative day, the uncorrected visual acuity (UCVA) of the right eye was 0.6 with clear cornea and slight inflammatory response in the anterior chamber. The patient's last visit was 3 months after surgery when the UCVA was 0.6 in the right eye with a clear cornea, a deep anterior chamber, and a stable IOL. The intraocular pressure was $14.5 \mathrm{mmHg}$ in the right eye. A retinal examination showed extensive old vasculitic changes.

\subsection{Case 2}

A 86-year-old man had ankylosing spondylitis for the past 38 years. Since 1976, his mobility had deteriorated gradually with progression of his kyphosis, stiffness of the low back, and contractures of the hips and knees. The patient had a very long history of bilateral chronic uveitis, with a visual acuity of hand movements only with accurate light projection in both eyes. Intraocular pressure, also measured with a Tono-Pen, was $12.4 \mathrm{mmHg}$ in the right eye and $15.2 \mathrm{mmHg}$ in the left eye. He had bilateral dense cataracts with extensive posterior synechiae, making the fundus examination impossible.

Then the right eye underwent phacoemulsification and a +19.5D intraocular lens (OII, Ophthalmic Innovation International, INC) implanted uneventfully with the same procedure. The effective phaco time for the right eye was 2'39", and the mean of $19 \%$ of ultrasound power. On the first postoperative day, the uncorrected visual acuity (UCVA) of the right eye was 0.4 with a clear cornea and no inflammatory response in the anterior chamber. The patient's last visit was 3 months after surgery when the UCVA was 0.5 in the right eye, with a clear cornea, a deep anterior chamber, a stable IOL, and normal intraocular pressure. 


\section{Discussion}

In 1994, Rimmer and Miller [3] reported performing phacoemulsification in a standing position for a patient unable to lie flat because of myotonic dystrophy, cervical kyphosis, and severe dyspnea. Recently, Mansour and Al-Dairy [2] suggested standing phacoemulsification for the patient in the reverse trendelenburg position, specifically to reduce the high rate of posterior capsule rupture in similar patients. We reported two cases in which combined clear corneal temporal phacoemulsification and foldable intraocular lens (IOL) implantation were performed using topical anesthesia with the patient's head positioned at a 60 degree inclination because of fixed cervical kyphosis without complications. To our knowledge, this is first time to report using topical anesthesia for standing phacoemulsification and intraocular lens implantaton for patients with marked cervical kyphosis by temporal approach. Ang et al. [4] operated successfully by face-to-face seated positioning for phacoemulsification in patients unable to lie flat for cataract surgery, but they used topical-intracameral anesthesia. Topical anesthesia is both a safe and effective method for smallincision clear corneal phacoemulsification cataract surgery, especially for patients who are old and have very high risk of cardiopulmonary complications for general anesthesia [5]. However, patients' cooperation is essential in surgery under topical anesthesia which usually is difficult for nervous patients. General anesthesia should be used in the patients who cannot cooperate and otherwise are in good health. On the other hand, it should be born in mind that incidence of perioperative systemic complications may increase in general anesthesia than local anesthesia.

Our two patients with long-standing ankylosing spondylitis and chronic uveitis needed cataract extraction. They presented a special situation as their extensive deformities resulted in a position in which the normal operating position at the head end was not feasible. Liu [6] noted surgical difficulties in a patient with torticollis, although the surgeon's position was not stated. However, the standing position was suggested if necessary. Fine et al. [7] reported altering a waiting room chair to permit adequate positioning of patients unable to lie flat on conventional operating tables, allowing surgery to be performed seated. The use of an orthopedic table allowed different segments of the spine and limbs to be positioned in different planes. The awkward angle of approach for the surgeon and the operating microscope resulted in difficulty focusing and in manipulating instruments and tissues. This was rendered manageable by carefully positioning the patient, the head in particular, on the orthopedic operating table, which allowed the pa- tient's head to be reclined to a position of 60 degrees to the horizontal. Successful combined phacoemulsification and foldable IOL implantation was then performed, although the angle of approach for the surgeon and the operating microscope was awkward. Caution must be used in elderly patients and those with cardiovascular compromise as a position with the feet raised much higher than the head may not be tolerated.

In exceptional circumstances, it is even possible to perform phacoemulsification with the patient's head in a semiupright position and the surgeon facing the patient. Our experience highlighted an strategy for unusual patients with severe kyphosis diseases in which surgeons had to perform using topical anesthesia for standing phacoemulsification and intraocular lens implantaton for patients with marked cervical kyphosis.

\section{Financial Disclosure}

The authors have no financial or proprietary interest in any materials or methods described herein. No conflicting relationship exists for any author.

\section{REFERENCES}

[1] S. Prasad, G. G. Kamath and R. P. Phillips, "Phacoemulsification in a Patient with Marked Cervical Kyphosis," Journal of Cataract \& Refractive Surgery, Vol. 26, No. 8, 2000, pp. 1258-1260. doi:10.1016/S0886-3350(00)00596-4

[2] A. M. Mansour and M. Al-Dairy, "Modifications in Cataract Surgery for the Morbidly Obese Patient," Journal of Cataract \& Refractive Surgery, Vol. 30, No. 11, 2004, pp. 2265-2268. doi:10.1016/j.jcrs.2004.02.088

[3] S. Rimmer and K. M. Miller, "Phacoemulsification in the Standing Position with Loupe Magnification and Headlamp Illumination,” Journal of Cataract \& Refractive Surgery, Vol. 20, No. 3, 1994, pp. 353-354.

[4] G. S. Ang, J. M. Ong and T. Eke, "Face-to-Face Seated Positioning for Phacoemulsification in Patients Unable to Lie Flat for Cataract Surgery,” American Journal of Ophthalmology, Vol. 141, No. 6, 2006, pp. 1151-1152. doi:10.1016/j.ajo.2006.01.036

[5] T. Stupp, I. Hassouna, K. Soppart, S. Thanos and W. Förster, "Systemic Adverse Events: A Comparison between Topical and Peribulbar Anesthesia in Cataract Surgery,” Ophthalmologica, Vol. 221, No. 5, 2007, pp. 320-325. doi:10.1159/000104762

[6] C. Liu, "Phacoemulsification in a Patient with Torticollis," Journal of Cataract \& Refractive Surgery, Vol. 21, No. 4, 1995, p. 364.

[7] I. H. Fine, R. S. Hoffman and S. Binstock, "Phacoemulsification Performed in a Modified Waiting Room Chair," Journal of Cataract \& Refractive Surgery, Vol. 22, No. 10, 1996, pp. 1408-1410. 